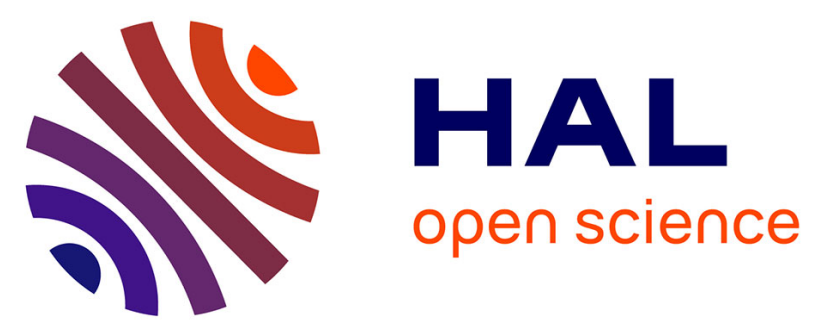

\title{
Isolation and characterisation of 26 microsatellite loci from a widespread tropical hydrozoan, Macrorhynchia phoenicea (Leptothecata, Aglaopheniidae), and cross-amplification in closely related species
}

\author{
Bautisse Postaire, D. Aurelle, Chloé A.-F. Annie-France Bourmaud, J.
} Henrich Bruggemann, Hélène Magalon

\section{To cite this version:}

Bautisse Postaire, D. Aurelle, Chloé A.-F. Annie-France Bourmaud, J. Henrich Bruggemann, Hélène Magalon. Isolation and characterisation of 26 microsatellite loci from a widespread tropical hydrozoan, Macrorhynchia phoenicea (Leptothecata, Aglaopheniidae), and cross-amplification in closely related species. Biochemical Systematics and Ecology, 2015, 62, 10.1016/j.bse.2015.08.011 . hal-01253779

\author{
HAL Id: hal-01253779 \\ https://hal.science/hal-01253779
}

Submitted on 26 Apr 2016

HAL is a multi-disciplinary open access archive for the deposit and dissemination of scientific research documents, whether they are published or not. The documents may come from teaching and research institutions in France or abroad, or from public or private research centers.
L'archive ouverte pluridisciplinaire HAL, est destinée au dépôt et à la diffusion de documents scientifiques de niveau recherche, publiés ou non, émanant des établissements d'enseignement et de recherche français ou étrangers, des laboratoires publics ou privés. 


\title{
Isolation and characterisation of 26 microsatellite loci from a widespread tropical hydrozoan, Macrorhynchia phoenicea (Leptothecata, Aglaopheniidae), and cross-amplification in closely related species
}

\author{
B. Postaire ${ }^{\mathrm{a}, \mathrm{b}, *, 1}$, D. Aurelle ${ }^{\mathrm{c}}$, C.A.F. Bourmaud ${ }^{\mathrm{a}, \mathrm{b}, 1}$, J.H. Bruggemann ${ }^{\mathrm{a}, \mathrm{b}, 1}$, \\ H. Magalon ${ }^{\mathrm{a}, \mathrm{b}, 1}$ \\ a Université de La Réunion UMR ENTROPIE 9220, CS 92003, 97744 Saint Denis CEDEX 9, France \\ ${ }^{\mathrm{b}}$ Laboratoire d'Excellence CORAIL, France \\ c Aix Marseille Université, CNRS, IRD, Avignon Université, IMBE UMR 7263, 13397 Marseille, France
}

\begin{abstract}
A B S T R A C T
We isolated and characterized 26 microsatellite loci for Macrorhynchia phoenicea (Busk, 1852), a rather common tropical hydrozoan from the Indo-Pacific. The number of alleles per locus ranged from 4 to 24 . The observed heterozygosity ranged from 0.000 to 0.970 and the expected heterozygosity from 0.029 to 0.833 . Ten loci were at Hardy-Weinberg equilibrium. No pair of loci presented linkage disequilibrium. Transferability of up to 18 loci was positive across four other Aglaopheniidae species from different genera. These loci will be used in studying reef population connectivity for these particular species at the scale of the Indo-Pacific, a promising but little explored research field.
\end{abstract}

\section{Introduction}

Hydrozoans are one of the most diverse marine suspension feeders, characterized by a wide range of reproductive modes and life cycles (Bouillon et al., 2006). While it is known that life history traits may affect population connectivity levels and diversification patterns (Schroth et al., 2002), this aspect has rarely been explored in hydroids, so that population genetics tools and relative data remain scarce for these organisms. To our knowledge, the only study of population genetics of hydrozoans using microsatellites focused on a freshwater invasive species in the Great Lakes region (USA) (Darling and FolinoRorem, 2009).

\footnotetext{
* Corresponding author. Université de La Réunion, Faculté des Sciences et Technologies, UMR ENTROPIE 9220, 15 Avenue René Cassin, CS 92003,97744 Saint Denis CEDEX 9, France.

E-mail address: bautisse.postaire@univ-reunion.fr (B. Postaire).

1 www.labex-corail.fr.
} 
The Aglaopheniidae is one of the most diverse family of feather-like hydroids, comprising more than 250 valid species, some of those presenting wide geographical distribution ranges, from temperate to tropical seas or from the surface to several hundreds of meters depth (Bouillon et al., 2006). Useful information on evolutionary processes and dispersal capacities of marine species can be generated using co-dominant neutral molecular markers such as microsatellites (Donald et al., 2011). However, while well represented and largely distributed on coral reefs, microsatellite markers in Aglaopheniidae have only been developed, to date, on a single one (Lytocarpia brevirostris (Busk, 1852); Postaire et al., 2015).

Here we describe the isolation of 26 microsatellite markers for Macrorhynchia phoenicea (Busk, 1852), a common tropical hydroid of this family, measuring several centimeters, inhabiting tropical coral reefs from South Pacific islands to Africa, from 2 to 70 m depth (Millard, 1975). Furthermore, we tested these new markers on four phylogenetically closely related species: Macrorhynchia philippina (Kirchenpauer, 1872), Macrorhynchia sibogae (Billard, 1913), Macrorhynchia spectabilis (Allman, 1883) and Lytocarpia nigra (Nutting, 1905). Studying the genetic diversity of these species and their populations' structure is crucial for assessing coral reefs biodiversity and conservation planning, as little is know on the extent of cryptic speciation and levels of endemism among these organisms.

\section{Material and methods}

\subsection{Construction of a microsatellite-enriched library and primer selection}

Total genomic DNA of nine individuals (whole colonies cleared from reproductive structures) from Reunion Island was isolated using DNeasy Blood \& Tissue kit $\left(\right.$ Qiagen $^{\mathrm{TM}}$ ) following manufacturer's protocol and sent to GenoScreen, Lille, France (www.genoscreen.fr). One $\mu \mathrm{g}$ was used for the development of microsatellites libraries through 454 GS-FLX Titanium pyrosequencing of enriched DNA libraries as described in Malausa et al. (2011). A total of 18,530 reads were obtained, of which 2958 contained microsatellite motifs. Obtained sequences were analyzed with QDD (Meglécz et al., 2010). Among the 204 primer pairs designed to amplify fragments containing a microsatellite motif, and on the basis on our criteria (PCR product $>100$ bp and number of repeats $\geq 6$ ), we selected 96 pairs of primers and tested their polymorphism on 16 individuals (eight from Reunion Island and eight from New Caledonia). Forward primers were indirectly fluorochrome labeled (6-FAM) by adding a 19-bp M13 tail at their $5^{\prime}$ end ( $5^{\prime}$ - CACGACGTTGTAAAACGAC-3') (Schuelke, 2000). Each amplification reaction was performed in a total volume of $10 \mu \mathrm{L}: 5 \mu \mathrm{L}$ of MasterMix Applied 2x (Applied Biosystems), $0.25 \mu \mathrm{L}$ ( $1 \mu \mathrm{M}$ ) of forward primer tagged with the M13 tail (final concentration: $0.025 \mu \mathrm{M}), 0.25 \mu \mathrm{L}(10 \mu \mathrm{M}$ ) of reverse primer (final concentration: $0.25 \mu \mathrm{M}$ ), $0.25 \mu \mathrm{L}(10 \mu \mathrm{M})$ of fluorescent dyed M13 tail (final concentration: $0.25 \mu \mathrm{M})$ and $2 \mu \mathrm{L}(10 \mathrm{ng} / \mu \mathrm{L})$ of genomic DNA (final concentration: $2 \mathrm{ng} / \mu \mathrm{L})$. The thermocycling program was as follows: $94{ }^{\circ} \mathrm{C}$ for $5 \mathrm{~min}+7 \mathrm{x}\left(94{ }^{\circ} \mathrm{C}\right.$ for $30 \mathrm{~s}, 62^{\circ} \mathrm{C}\left[-1{ }^{\circ} \mathrm{C}\right.$ at each cycle] for $30 \mathrm{~s}, 72{ }^{\circ} \mathrm{C}$ for $\left.30 \mathrm{~s}\right)+30 \mathrm{x}\left(94^{\circ} \mathrm{C}\right.$ for $30 \mathrm{~s}, 55^{\circ} \mathrm{C}$ for $30 \mathrm{~s}, 72{ }^{\circ} \mathrm{C}$ for $\left.30 \mathrm{~s}\right)+8 \mathrm{x}\left(94{ }^{\circ} \mathrm{C}\right.$ for $30 \mathrm{~s}, 56{ }^{\circ} \mathrm{C}$ for $30 \mathrm{~s}, 72{ }^{\circ} \mathrm{C}$ for $30 \mathrm{~s}$ ) for the final hybridization of fluorescent dyed M13 tail $+72{ }^{\circ} \mathrm{C}$ for $5 \mathrm{~min}$. PCR products were genotyped using an ABI 3130 genetic analyzer (Applied Biosystems) and allelic sizes were determined using GeneMapper v4.0 (Applied Biosystems). Primer pairs were eliminated from further development when (1) they failed to amplify, (2) they amplified multiple fragments, or (3) genotype scoring was deemed unreliable. Finally, a total of 26 candidate loci were successfully kept for characterizing polymorphism on a single population.

\subsection{Polymorphism testing and microsatellite cross-amplification}

Genotyping of these 26 markers was conducted on 34 individuals from a M. phoenicea population from Reunion Island (Saint Pierre; 21.35015 S, 55.48202 E) and, to explore allelic richness at large geographic scale, on individuals from Juan de Nova Island (Scattered Islands; $n=16)$, Madagascar $(n=16)$ and New Caledonia $(n=16)$ using the same protocol as above. A different dye was assigned to each locus (6-FAM, VIC, NED, PET) and PCR products were pooled in panels according to amplified fragment sizes and dyes to maximize efficiency and minimize costs. Cross-amplifications of these 26 markers were also tested using the same protocol on M. philippina, M. sibogae, M. spectabilis and L. nigra.

\subsection{Data analysis}

Allele sizes and number of alleles per locus are indicated for the whole dataset (i.e. all sampling sites from South Western Indian Ocean and Pacific); population statistics were calculated for the Reunion Island population only ( $\mathrm{n}=34)$. Diversity indices were assessed and conformation to Hardy-Weinberg expectations tested using Arlequin v 3.5.1.2 (Excoffier et al., 2005). Tests of linkage disequilibrium between all pairs of loci were conducted using Genepop v 4.2. Null alleles and other potential technical biases were tested using Micro-Checker v2.2.3 (van Oosterhout et al., 2004).

\section{Results and discussion}

All 26 loci were polymorphic. From the genotyping of the Indo-Pacific sampling, the number of alleles per locus ranged from 4 to 24; in the tested population from Reunion Island, the number of alleles ranged from 2 to 7 . Observed heterozygosities in the Reunion Island population ranged from 0.000 to 0.970 and expected heterozygosities from 0.029 to 0.833 (Table 1). Over 325 linkage disequilibrium tests between all pairs of loci, only 16 were significant after False Discovery Rate 
Table 1

Microsatellite loci developed for Macrorhynchia phoenicea and their primer sequences (F: forward, R: reverse). Accession numbers in GenBank are indicated. Diversity indices are issued from Arlequin v3.5.1.2. $N_{a}$ : number of alleles per locus at the Indo-Pacific scale; $N_{a}$ pop: number of alleles per locus in the tested population (Reunion Island); $N$ : number of genotyped individuals; $H_{O}$ : observed heterozygosity; $H_{E}$ : expected heterozygosity; $F_{I S}$ : inbreeding coefficient. Under the $F_{I S}$ are indicated the significance of the $P$-values for deviation to Hardy-Weinberg equilibrium (1000 permutations): NS: non significant; *: $P<0.05 ;^{* *}: P<0.01 ;^{* * *}: P<0.001$. Null allele frequencies $(r)$ are issued from Micro-Checker v 2.2.3 (van Oosterhout et al., 2004 ), and presence or absence is indicated below (yes or no).

\begin{tabular}{|c|c|c|c|c|c|c|c|c|c|c|c|c|c|}
\hline $\begin{array}{l}\text { Locus } \\
\text { name }\end{array}$ & Primer sequence $\left(5^{\prime}-3^{\prime}\right)$ & $\begin{array}{l}\text { GenBank } \\
\text { accession } \\
\text { number }\end{array}$ & $\begin{array}{l}\text { Repeat } \\
\text { motif }\end{array}$ & Dye & Panel & Size (bp) & $N_{a}$ & $N_{a p o p}$ & $N$ & $H_{O}$ & $H_{E}$ & $F_{I S}$ & $r$ \\
\hline Mp01 & $\begin{array}{l}\text { F: GGCAACTAACAACCGCATTT } \\
\text { R: AATAAGTCAGAAGCATTATGGTCAA }\end{array}$ & KM675787 & $(\mathrm{TATC})_{13}$ & VIC & 1 & $99-170$ & 16 & 7 & 34 & 0.206 & 0.833 & $\begin{array}{l}0.756 \\
* * *\end{array}$ & $\begin{array}{l}0.360 \\
\text { yes }\end{array}$ \\
\hline Mp02 & $\begin{array}{l}\text { F: CCAATAACATGCATGCAACA } \\
\text { R: TCGAACCTTGTGTCCTTGTG }\end{array}$ & KM675788 & $(\mathrm{CAA})_{9}$ & 6-FAM & 1 & $106-133$ & 8 & 2 & 34 & 0.029 & 0.029 & $\begin{array}{l}0.000 \\
\text { NS }\end{array}$ & $\begin{array}{l}-0.014 \\
\text { no }\end{array}$ \\
\hline Mp03 & $\begin{array}{l}\text { F: GGTTCAAATTAAAAGACGTCCC } \\
\text { R: TCCTAACCCTTTTCTTACCCC }\end{array}$ & KM675789 & $(\mathrm{ACA})_{7}$ & NED & 1 & $153-194$ & 13 & 2 & 34 & 0.029 & 0.275 & $\begin{array}{l}0.895 \\
* * *\end{array}$ & $\begin{array}{l}0.291 \\
\text { yes }\end{array}$ \\
\hline Mp04 & $\begin{array}{l}\text { F: CTTCCAGCCATGAAAGTTGA } \\
\text { R: ATACGCTCCTGGATTGGTTG }\end{array}$ & KM675790 & $(\mathrm{CAA})_{7}$ & PET & 1 & $150-189$ & 14 & 2 & 34 & 0.000 & 0.506 & $\begin{array}{l}1.000 \\
* * *\end{array}$ & $\begin{array}{l}0.414 \\
\text { yes }\end{array}$ \\
\hline Mp05 & $\begin{array}{l}\text { F: TGCTAATTATAGACAGGTTACATTTGA } \\
\text { R: TTGGGCTAGGTAACATTACCACT }\end{array}$ & KM675791 & $(\mathrm{TAGC})_{5}$ & 6-FAM & 1 & $228-256$ & 6 & 3 & 34 & 0.970 & 0.534 & $\begin{array}{l}-0.840 \\
* * *\end{array}$ & $\begin{array}{l}-0.733 \\
\text { no }\end{array}$ \\
\hline Mp06 & $\begin{array}{l}\text { F: CGAGAATTCCACCCAATG } \\
\text { R: CGACGATTTACTACAAGTCCAAGA }\end{array}$ & KM675792 & $(\mathrm{TGT})_{11}$ & VIC & 1 & $268-333$ & 17 & 4 & 34 & 0.029 & 0.244 & $\begin{array}{l}0.881 \\
* * *\end{array}$ & $\begin{array}{l}0.268 \\
\text { yes }\end{array}$ \\
\hline Mp07 & $\begin{array}{l}\text { F: ATGCGTATGCAAAGGATTGG } \\
\text { R: AAGGAGGCAGTTGCTATGGT }\end{array}$ & KM675793 & $(\mathrm{GA})_{9}$ & 6-FAM & 2 & $140-164$ & 10 & 3 & 34 & 0.029 & 0.086 & $\begin{array}{l}0.663 \\
\text { NS }\end{array}$ & $\begin{array}{l}0.142 \\
\text { yes }\end{array}$ \\
\hline Mp08 & $\begin{array}{l}\text { F: GTCAAGTTGGTTTCACTCATGC } \\
\text { R: ACAAGCAACGATGAAGGGAC }\end{array}$ & KM675794 & $(\mathrm{GTT})_{7}$ & VIC & 2 & $155-167$ & 10 & 3 & 34 & 0.323 & 0.586 & $\begin{array}{l}0.452 \\
* * *\end{array}$ & $\begin{array}{l}0.231 \\
\text { yes }\end{array}$ \\
\hline Mp09 & $\begin{array}{l}\text { F: ATTGATTCACGCTTGTGCAG } \\
\text { R: GGTTGTTAGATCAGGTAATGTGG }\end{array}$ & KM675795 & $(\mathrm{AG})_{7}$ & NED & 2 & $176-196$ & 10 & 2 & 34 & 0.059 & 0.058 & $\begin{array}{l}-0.015 \\
\text { NS }\end{array}$ & $\begin{array}{l}-0.030 \\
\text { no }\end{array}$ \\
\hline Mp10 & $\begin{array}{l}\text { F: TTATCCCGCTAAACATGAAACA } \\
\text { R: CGCGTTATGTAGATCAGCCA }\end{array}$ & KM675796 & $(\text { CGTTGT })_{5}$ & 6-FAM & 2 & $236-347$ & 24 & 3 & 34 & 0.000 & 0.262 & $\begin{array}{l}1.000 \\
* * *\end{array}$ & $\begin{array}{l}0.308 \\
\text { yes }\end{array}$ \\
\hline Mp11 & $\begin{array}{l}\text { F: GGAAAACGACCACAATCCAA } \\
\text { R: CTCTTGATTTGATTCAGGAATGT }\end{array}$ & KM675797 & $(\mathrm{CTAT})_{9}$ & VIC & 2 & $289-338$ & 10 & 3 & 34 & 0.235 & 0.643 & $\begin{array}{l}0.638 \\
* * *\end{array}$ & $\begin{array}{l}0.288 \\
\text { yes }\end{array}$ \\
\hline Mp12 & $\begin{array}{l}\text { F: CGAGTCGTTGCAGGGTTACT } \\
\text { R: TCGACGTTATATCTCAAACCTCA }\end{array}$ & KM675798 & $(\mathrm{AGAT})_{7}$ & VIC & 3 & $135-223$ & 21 & 3 & 34 & 0.088 & 0.319 & $\begin{array}{l}0.727 \\
* * *\end{array}$ & $\begin{array}{l}0.260 \\
\text { yes }\end{array}$ \\
\hline Mp13 & $\begin{array}{l}\text { F: CTTCACCGCTATTAGCTCTTCA } \\
\text { R: TTCATAGCGATACGAAACACG }\end{array}$ & KM675799 & $(\mathrm{GA})_{7}$ & 6-FAM & 3 & $138-164$ & 8 & 2 & 34 & 0.000 & 0.395 & $\begin{array}{l}1.000 \\
* * *\end{array}$ & $\begin{array}{l}0.372 \\
\text { yes }\end{array}$ \\
\hline Mp14 & $\begin{array}{l}\text { F: TTTCTCCAAATTGTTCCAAGC } \\
\text { R: GCAAATGCCTAAGCTGCAA }\end{array}$ & KM675800 & $(\mathrm{GTT})_{6}$ & NED & 3 & $177-204$ & 7 & 2 & 34 & 0.000 & 0.058 & $\begin{array}{l}1.000 \\
\text { NS }\end{array}$ & $\begin{array}{l}0.157 \\
\text { yes }\end{array}$ \\
\hline Mp15 & $\begin{array}{l}\text { F: GAGGCGTTTCCTTTTCAAAT } \\
\text { R: GAACGTTTCCTCGGAGTGAC }\end{array}$ & KM675801 & $(\mathrm{CAA})_{9}$ & PET & 3 & $202-228$ & 9 & 3 & 34 & 0.176 & 0.388 & $\begin{array}{l}0.548 \\
* * *\end{array}$ & $\begin{array}{l}0.228 \\
\text { yes }\end{array}$ \\
\hline Mp16 & $\begin{array}{l}\text { F: TCAGAGTTCAAGGGCCAAGT } \\
\text { R: TGGGAAGTGTGACCTCGATT }\end{array}$ & KM675802 & $(\mathrm{AAC})_{6}$ & 6-FAM & 3 & $197-218$ & 6 & 2 & 34 & 0.029 & 0.187 & $\begin{array}{l}0.845 \\
* * *\end{array}$ & $\begin{array}{l}0.236 \\
\text { yes }\end{array}$ \\
\hline Mp17 & $\begin{array}{l}\text { F: GCTTTCTCAACAGTTTTCTATTGG } \\
\text { R: CGTGTGAGCCTTTCACTTGAT }\end{array}$ & KM675803 & $(\mathrm{TG})_{9}$ & 6-FAM & 4 & $114-144$ & 15 & 6 & 34 & 0.618 & 0.798 & $\begin{array}{l}0.228 \\
* * *\end{array}$ & $\begin{array}{l}0.096 \\
\text { yes }\end{array}$ \\
\hline Mp18 & $\begin{array}{l}\text { F: TCCCACTGCGTTGTATTGTG } \\
\text { R: GGAAATAATTGGTTTCCAGACAA }\end{array}$ & KM675804 & $(\mathrm{ATCT})_{6}$ & VIC & 4 & $127-180$ & 16 & 6 & 34 & 0.588 & 0.727 & $\begin{array}{l}0.194 \\
\text { NS }\end{array}$ & $\begin{array}{l}0.077 \\
\text { no }\end{array}$ \\
\hline Mp19 & $\begin{array}{l}\text { F: CAGACATAAATTACGAATAATTAACCA } \\
\text { R: CCTGCTTTTCTCTTTTGCTTTC }\end{array}$ & KM675805 & $(\mathrm{GT})_{9}$ & NED & 4 & $153-174$ & 5 & 3 & 34 & 0.412 & 0.505 & $\begin{array}{l}0.218 \\
\text { NS }\end{array}$ & $\begin{array}{l}0.097 \\
\text { no }\end{array}$ \\
\hline Mp20 & $\begin{array}{l}\text { F: CGCTGACCAGTCCGTTTAAT } \\
\text { R: GTTGAAGTTCACCAACGGCT }\end{array}$ & KM675806 & $(\mathrm{GTT})_{6}$ & PET & 4 & $192-204$ & 5 & 2 & 34 & 0.029 & 0.029 & $\begin{array}{l}0.000 \\
\text { NS }\end{array}$ & $\begin{array}{l}-0.015 \\
\text { no }\end{array}$ \\
\hline Mp21 & $\begin{array}{l}\text { F: TCCGTTGTGTCTATGATATGTTCTT } \\
\text { R: CCACATGGTTATCGTCAGCA }\end{array}$ & KM675807 & $(\mathrm{TTG})_{7}$ & 6-FAM & 4 & $271-315$ & 16 & 4 & 34 & 0.265 & 0.331 & $\begin{array}{l}0.204 \\
\text { NS }\end{array}$ & $\begin{array}{l}0.072 \\
\text { no }\end{array}$ \\
\hline Mp22 & $\begin{array}{l}\text { F: TTCGCTCTTTCATTCATCCG } \\
\text { R: GAGAAAAGAAATGAAACGCACA }\end{array}$ & KM675808 & $(\mathrm{TG})_{6}$ & 6-FAM & 5 & $119-129$ & 6 & 4 & 34 & 0.176 & 0.518 & $\begin{array}{l}0.663 \\
* * *\end{array}$ & $\begin{array}{l}0.287 \\
\text { yes }\end{array}$ \\
\hline Mp23 & $\begin{array}{l}\text { F: GGCGACGAAGATTTGAGAAC } \\
\text { R: GAGTGTGGATGTTGCGTGAG }\end{array}$ & KM675809 & $(\mathrm{AAC})_{12}$ & VIC & 5 & $126-168$ & 15 & 4 & 34 & 0.353 & 0.347 & $\begin{array}{l}-0.017 \\
\text { NS }\end{array}$ & $\begin{array}{l}-0.036 \\
\text { no }\end{array}$ \\
\hline Mp24 & $\begin{array}{l}\text { F: AATACGGCGTCAGAAACCTC } \\
\text { R: GCGATCCACCAAATGCTTAT }\end{array}$ & KM675810 & $(\mathrm{CT})_{8}$ & NED & 5 & $136-164$ & 24 & 4 & 34 & 0.118 & 0.528 & $\begin{array}{l}0.780 \\
* * *\end{array}$ & $\begin{array}{l}0.331 \\
\text { yes }\end{array}$ \\
\hline Mp25 & $\begin{array}{l}\text { F: CACTGGACTACGATCACTCATTG } \\
\text { R: AGGAGACGAGAATGTCGGAA }\end{array}$ & KM675811 & $(\mathrm{TGT})_{7}$ & PET & 5 & 157-169 & 4 & 3 & 34 & 0.059 & 0.437 & $\begin{array}{l}0.867 \\
* * *\end{array}$ & $\begin{array}{l}0.340 \\
\text { yes }\end{array}$ \\
\hline Mp26 & $\begin{array}{l}\text { F: CAAGATCAAGGACACCGAGC } \\
\text { R: TCAAATCATCGCCACTAGACA }\end{array}$ & KM675812 & $(\mathrm{CAA})_{6}$ & 6-FAM & 5 & $219-242$ & 9 & 2 & 34 & 0.000 & 0.058 & $\begin{array}{l}1.000 \\
\text { NS }\end{array}$ & $\begin{array}{l}0.157 \\
\text { yes }\end{array}$ \\
\hline
\end{tabular}

(FDR) correction for multiple testing (Benjamini and Hochberg, 1995). In the tested population, 10 loci over 26 were at Hardy-Weinberg equilibrium. All the other loci showed significant heterozygotes deficit ( $F_{I S}$ ranging from 0.228 to 1 ) except Mp05 $\left(F_{I S}=-0.84\right)$. On one hand, this is potentially due to the presence of null alleles, revealed using Micro-Checker v2.2.3 (van Oosterhout et al., 2004, Table 1). However, no null homozygotes were found, indicating that if null alleles are present, they may occur in low frequencies. Significant heterozygotes deficit, on the other hand, is consistent with mating between related individuals for these loci and might reflect the specific sexuality (gonochoric) and mode of larval development of this species. Indeed, like the majority of Aglaopheniidae, M. phoenicea is a brooder species, incubating its larvae in dedicated 
Table 2

Cross-amplification for 26 microsatellite markers designed for Macrorhynchia phoenicea across four Aglaopheniidae species: Lytocarpia nigra ( $\mathrm{n}=6$ ), M. philippina $(\mathrm{n}=10)$, M. sibogae $(\mathrm{n}=1)$ and $M$. spectabilis $(\mathrm{n}=1)(+$, amplified; + P, polymorphic; - , no amplification). Size ranges of the PCR product in base pairs (19 bp M13 tail included) and number of alleles (in brackets) are also indicated.

\begin{tabular}{|c|c|c|c|c|}
\hline Locus Name & L. nigra $(\mathrm{n}=6)$ & M. philippina $(\mathrm{n}=10)$ & M. sibogae $(\mathrm{n}=1)$ & M. spectabilis $(\mathrm{n}=1)$ \\
\hline Mp01 & - & - & - & - \\
\hline Mp02 & - & - & - & - \\
\hline Mp03 & $+\mathrm{P} 162-165(2)$ & $+\mathrm{P} 162-165(2)$ & - & $+\mathrm{P} 165-168$ \\
\hline Mp04 & $+162(1)$ & $+\mathrm{P} 159-162(2)$ & - & $+\mathrm{P} 162-174(2)$ \\
\hline Mp05 & + P 230-256 (3) & + P 230-256 (4) & - & $+\mathrm{P} 232-240(2)$ \\
\hline Mp06 & $+\mathrm{P} 305-311(3)$ & +P 292-311 (3) & - & $+\mathrm{P} 305-311(2)$ \\
\hline Mp07 & $+140(1)$ & $+\mathrm{P} 144-150$ & - & $+148(1)$ \\
\hline Mp08 & $+\mathrm{P} 159-167$ (4) & $+\mathrm{P} 155-167$ (5) & - & $+\mathrm{P} 161-165$ \\
\hline Mp09 & $+\mathrm{P} 186-188(2)$ & $+\mathrm{P} 186-188$ & $+188(1)$ & $+186(1)$ \\
\hline Mp10 & $+253(1)$ & + P 253-269 (4) & - & $+253(1)$ \\
\hline Mp11 & - & - & - & - \\
\hline Mp12 & - & - & - & - \\
\hline Mp13 & $+\mathrm{P} 154-160(2)$ & $+160(1)$ & - & $+162(1)$ \\
\hline Mp14 & $+182(1)$ & $+182(1)$ & - & $+182(1)$ \\
\hline Mp15 & + P 210-225 (4) & $+\mathrm{P} 213-225$ & - & $+\mathrm{P} 208-216(2)$ \\
\hline Mp16 & $+212(1)$ & $+\mathrm{P} 212-215$ & - & $+212(1)$ \\
\hline Mp17 & $+\mathrm{P} 130-134(2)$ & $+\mathrm{P} 124-134(4)$ & - & $+\mathrm{P} 128-130(2)$ \\
\hline Mp18 & $+144(1)$ & - & - & - \\
\hline Mp19 & - & +159(1) & - & $+\mathrm{P} 164-178(2)$ \\
\hline Mp20 & $+\mathrm{P} 198-201(2)$ & +P 198-201 (2) & +P 198-201 (2) & $+201(1)$ \\
\hline Mp21 & - & $+291(1)$ & - & $+\mathrm{P} 272-275$ \\
\hline Mp22 & $+\mathrm{P} 121-129$ & $+\mathrm{P} 121-127(3)$ & $+\mathrm{P} 121-127(2)$ & $+121(1)$ \\
\hline \multirow[t]{2}{*}{ Mp23 } & $+\mathrm{P} 136-139(2)$ & $+\mathrm{P} 136-139(2)$ & & $+\mathrm{P} 132-142(2)$ \\
\hline & & & $+\mathrm{P} 136-139(2)$ & \\
\hline Mp24 & $+143(1)$ & $+\mathrm{P} 141-145$ & - & $+\mathrm{P} 154-156(2)$ \\
\hline Mp25 & - & - & - & $+169(1)$ \\
\hline Mp26 & $+\mathrm{P} 222-233(4)$ & $+\mathrm{P} 227-236(2)$ & $+236(1)$ & $+233(1)$ \\
\hline
\end{tabular}

structures. When mature, released planulae usually crawl (rather than swim) for a short distance until metamorphosis, which usually starts within less than a day (Sommer, 1990). The only true planktonic phase of their life cycle corresponds to the release of spermatozoids in the water column, which life span has been studied for only one other hydroid species, and seems to be reduced to a few hours (Yund, 1990). In addition, Aglaopheniidae species do not seem to reproduce during mass spawning events like many scleractinians: in several species in Reunion Island coral reefs, the presence of reproductive structures have been observed during several months (CAFB pers. obs.). In temperate marine hydrozoans species, the reproduction period is related to environmental factors and consequently colonies in the same location tend to reproduce at the same time (Gili and Hughes, 1995). Thus fertilization occurs preferentially between geographically close individuals, which may be potentially related. All these aspects naturally contribute to low levels of genetic diversity within populations, as migration events are potentially rare. The assumption of reduced gene flow among populations will be further addressed in a population genetic study of this species with a broad geographical coverage, from South-Western Indian Ocean to Pacific.

Cross-species amplification tests revealed that various loci amplified across closely related species of this family (Table 2). Among the 26 loci, (1) 12 and 9 were polymorphic while amplifying $M$. philippina $(\mathrm{n}=10)$ and $L$. nigra $(\mathrm{n}=6)$, respectively; (2) M. spectabilis $(\mathrm{n}=1)$ and $M$. sibogae $(\mathrm{n}=1)$ were heterozygotes for 11 and 3 loci, respectively. This shows high transferability within the same genus, and between genera from the same family. These loci might be useful for other species within this family that have not been tested in this study. More generally, these loci will be useful in phylogeographic studies, potentially revealing biogeographic barriers, and will help to investigate relationships between larval dispersal capacities and gene flow of such coastal benthic species.

\section{Acknowledgments}

Hydrozoan sampling in the western lagoon of New Caledonia (HM) was carried out during Cobelo campaign onboard of RV Alis of IRD. Sampling in Reunion Island (CAFB, HB, HM, BP) was supported by program HYDROSOOI (Labex CORAIL fund). Sampling in Madagascar (HM) was supported by project Biodiversity (POCT FEDER fund) and sampling in Juan de Nova was supported by program Biorecie (POCT FEDER fund). We gratefully acknowledge the Plateforme Gentyane of the Institut National de la Recherche Agronomique (INRA, Clermont-Ferrand, France) for guidance and technical support. The first author was financially supported by a PhD contract from the Doctoral School of the University of Reunion Island.

\section{References}


Benjamini, Y., Hochberg, Y., 1995. Controlling the false discovery rate: a practical and powerful approach to multiple testing. J. Royal Stat. Soc. Ser. B Methodol. 57, 289-300 doi:10.2307/2346101.

Billard, A., 1913. Les hydroïdes de l'expédition du Siboga, i. Plumulariidae. Résultats de l'Exploration du Siboga 7, 1-115.

Bouillon, J., Gravili, C., Pagès, F., Gili, J.-M., Boero, F., 2006. An Introduction to Hydrozoa. Mémoires du Muséum d'Histoire Naturelle, Paris.

Busk, G., 1852. An account of the Polyzoa and sertularian zoophytes collected in the voyage of the "rattlesnake" on the coast of Australia and the Louisiade Archipelago. In: MacGillivray, J. (Ed.), Narrative of the Voyage of H.M.S. Rattlesnake, Commanded by the Late Captain Owen Stanley during the Years 1846-50. Boone, London, pp. 343-402.

Darling, J.A., Folino-Rorem, N.C., 2009. Genetic analysis across different spatial scales reveals multiple dispersal mechanisms for the invasive hydrozoan Cordylophora in the Great Lakes. Mol. Ecol. 18, 4827-4840. http://dx.doi.org/10.1111/j.1365-294x.2009.04405.x.

Donald, K.M., Keeney, D.B., Spencer, H.G., 2011. Contrasting population makeup of two intertidal gastropod species that differ in dispersal opportunities. J. Exp. Mar. Biol. Ecol. 396, 224-232. http://dx.doi.org/10.1016/j.jembe.2010.10.028.

Excoffier, L., Laval, G., Schneider, S., 2005. Arlequin (version 3.0): an integrated software package for population genetics data analysis. Evol. Bioinforma. 1, $47-50$

Gili, J.-M., Hughes, R.G., 1995. The ecology of marine benthic hydroids. Oceanogr. Mar. Biol. An Annu. Rev. $33,351-426$.

Kirchenpauer, G.H., 1872. Ueber die Hydroidenfamilie Plumularidae, einzelne Gruppen derselben und ihre Fruchtbehälter. I. Aglaophenia, Lx. Abh. Geb. Naturw. Hambourg. 5, 1-52 pls 1-8.

Malausa, T., Gilles, A., Meglécz, E., Blanquart, H., Duthoy, S., Costedoat, C., Dubut, V., Pech, N., Castgnone-Serrano, P., Délye, C., Feau, N., Frey, P., Gauthier, P., Guillemaud, T., Hazard, L., Le Corre, V., Lung-Escarmant, B., Malé, P.-J.G., Ferreira, S., Martin, J.-F., 2011. High-throughput microsatellite isolation through 454 GS-FLX Titanium pyrosequencing of enriched DNA libraries. Mol. Ecol. Resour. 11, 638-644. http://dx.doi.org/10.1111/j.1755-0998.2011.02992.x.

Meglécz, E., Costedoat, C., Dubut, V., Gilles, A., Malausa, T., Pech, N., Martin, J.-F., 2010. QDD: a user-friendly program to select microsatellite markers and design primers from large sequencing projects. Bioinformatics 26, 403-404. http://dx.doi.org/10.1093/bioinformatics/btp670.

Millard, N.A.H., 1975. Monograph on the Hydroida of Southern Africa. Kaapstad.

Nutting, C.C., 1905. Hydroids of the Hawaiian Islands collected by the steamer Albatross in 1902. Bull. U. S. Fish Comm. 23, 931-959.

Postaire, B., Aurelle, D., Bourmaud, C.A.F., Bruggemann, J.H., Magalon, H., 2015. Isolation and characterisation of 16 microsatellite loci from a widespread tropical hydrozoan, Lytocarpia brevirostris (Busk, 1852). Conserv. Genet. Resour. 7, 505-507. http://dx.doi.org/10.1007/s12686-014-0407-1.

Schroth, W., Jarms, G., Streit, B., Schierwater, B., 2002. Speciation and phylogeography in the cosmopolitan marine moon jelly, Aurelia sp. BMC Evol. Biol. $2,1$. http://dx.doi.org/10.1186/1471-2148-2-1.

Schuelke, M., 2000. An economic method for the fluorescent labeling of PCR fragments. Nat. Biotechnol. 18, 233-234. http://dx.doi.org/10.1038/72708.

Sommer, C., 1990. Post-embryonic larval development and metamorphosis of the hydroid Eudendrium racemosum (Cavolini) (Hydrozoa, Cnidaria). Helgol. Meeresunters. 44, 425-444. http://dx.doi.org/10.1007/BF02365478.

van Oosterhout, C., Hutchinson, W.F., Wills, D.P.M., Shipley, P., 2004. MICRO-CHECKER: software for identifying and correcting genotyping errors in microsatellite data. Mol. Ecol. Notes 4, 535-538. http://dx.doi.org/10.1111/j.1471-8286.2004.00684.x.

Yund, P.O., 1990. An in situ measurement of sperm dispersal in a colonial marine hydroid. J. Exp. Zool. 253, 102-106. http://dx.doi.org/10.1002/jez. 1402530114. 